\title{
Research Paper \\ Role of Performance in the Stroop Test in Anticipation of Anxiety and Aggression in the Elderly in Ardabil
}

\author{
*Esmaeil Sadri Damirchi ${ }^{1} \oplus$, Sanaz Behbuei ${ }^{2}$, Arezoo Mojarrad ${ }^{1}$
}

1. Department of Educational Sciences, Faculty of Education and Psychology, University of Mohaghegh Ardabili, Ardabil, Iran.

2. Department of Psychology, Faculty of Education and Psychology, University of Tabriz, Tabriz, Iran.

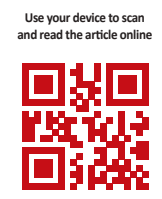

diteation: Sadri Damirchi E, Behbuei S, Mojarrad A. [Role of Performance in the Stroop Test in Anticipation of Anxiety and Aggression in the Elderly in Ardabil (Persian)]. Iranian Journal of Ageing. 2018; 13(1):38-49. https://doi.org/10.21859/SIJA.13.1.38

Received: 05 Sep. 2017

Accepted: 03 Jan. 2018

Key words:

Strop function,

Anxiety, Fall self-

efficacy, Elderly

\section{A B STRACT}

Objectives Currently, the life expectancy of the elderly has been rising considerably. The present study investigated the role of strop function in the prediction of anxiety and fall self-efficacy in elderly at the city of Ardabil.

Methods \& Materials The study design was correlational. The population cohort consisted of all elderly males living in the elderly homes in Ardabil in2017. Among these, a sample of 200 subjects was selected by the available sampling method. The anxiety, fall self-efficacy, and Stroop questionnaires were used for data collection. The data were analyzed by Pearson's correlation and regression analysis.

Results A significant relationship was observed between anxiety/fall self-efficacy and Strop function. Stroop's performance could negatively predict anxiety as well as predict the fall self-efficacy in the elderly. Conclusion The performance of Stroop test in the elderly could be improved by training classes and various treatment groups that can indirectly decrease the amount of anxiety and increase the fall self-efficacy in the elderly.

\section{Extended Abstract}

\section{Objectives}

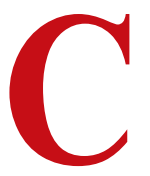

urrently, due to an increase in life expectancy, the old age phenomenon has been under intensive focus more than before due to the associated issues and dilemmas. Aging is an inevitable biological process involving all living beings, and in some cases, unpleasant experiences [1]. According to international estimates, since 1419, the elderly population of Iran has grown rapidly as compared to other locations as well as the average growth rate of the elderly worldwide. Until
1424 , Iran overtook the average rate of population growth of the elderly and surpassed that of Asia within the subsequent5 years [2]. Over the last 50 years, the proportion and number of elderly individuals have increased in a majority of the Third World countries [3]. Given the rising population of the elderly and the associated problems, the present study investigated the role of Stroop performance with respect to anxiety and self-efficacy in falling of the elderly $>60$ years of age.

\section{Methods and Materials}

The research design was descriptive correlational. The statistical population included all elderly men

Address: Department of Educational Sciences, Faculty of Education and Psychology, University of Mohaghegh Ardabili, Ardabil, Iran

E-mail: e.sadri@uma.ac.ir 
$>60$-year-old, who were residents of the nursing home of Ardebil city in 2017. Among these, a sample of 200 individuals was selected by the available sampling method. The present study was approved by the Welfare Organization of Ardebil Committee and conducted after coordination with the Welfare Organization of the city of Ardebil and Rayehe Omid Nursing Home; appropriate research and ethical permissions were obtained prior to the study. Data were analyzed using Pearson's correlation test and regression analysis. $\mathrm{P}<0.05$ was considered as statistically significant. The following questionnaires were used to collect the information:

\section{Stroop Test}

Stroop Test was invented in 1935 by Stroop to evaluate the proprietary attention and cognitive flexibility. Since then, several variations of this test have been available. The reliability of this test for the first and second cards was 0.81 , and that for the third and fourth cards was 0.80 [4].

\section{Beck Anxiety Inventory}

Beck Anxiety Inventory was introduced by Iron Beck et al. (1990). This questionnaire specifically measured the severity of the symptoms of clinical anxiety in individuals. This questionnaire contains 21 options. The coefficient of internal consistency (alpha coefficient) was 0.92 , and its validity by retest method at an interval of 1week was 0.75 [5]. Kaviani and Mousavi (1999) also reported the coefficient of the validity of this questionnaire as 0.72 and there-validation factor with a1-month interval as 0.83 ; the Cronbach's alpha coefficient was 0.92 [40].

\section{Falls Self-efficacy Scale (FES-I)}

Falls Self-efficacy Scale (FES-I) comprises of 16 items invented by Yardley et al. The items in this questionnaire had four options: "I am not at all worried" to "I am quite worried" and the score of each subject, will be the total points from 16 questions. The scores range was 16-64. A high score indicated fear of falling or low self-efficacy [6]. Furthermore, Borhani Nezhad et al. (2015) reported the Cronbach's alpha coefficient as 0.909 [43].

\section{Results}

Herein, 200 married elderly men $>60$-year-old [Mean $(\mathrm{SD})=47.66(6.576)]$ participated in the study. The de-

Table 1. Descriptive statistics of research variables

\begin{tabular}{cccc}
\hline & Components & Mean & Standard Deviation \\
\hline & Self-Efficacy in Falling & 58.5350 & 4.9559 \\
& Anxiety & 14.121 & 10.266 \\
& Correct & 24.965 & 0.232 \\
First card & Reaction & 22.815 & 7.113 \\
& Reaction & 0.576 & 3.592 \\
& Error & 2.595 & 6.579 \\
Cecond card & Reaction & 23.388 & 4.733 \\
& Reaction & 26.205 & 13.263 \\
& Error & 10.059 & 19.843 \\
& Correct & 0.740 & 1.849 \\
Third card & Reaction & 24.245 & 1.847 \\
& Reaction & 25.489 & 10.029 \\
& Error & 6.487 & 10.968 \\
& Correct & 3.070 & 4.521 \\
& Reaction & 21.900 & 4.514 \\
& Reaction & 49.472 & 54.784 \\
& Error & 37.2766 & 54.708 \\
& & 3.070 & 4.5218 \\
\hline
\end{tabular}


Table 2. Results of Pearson correlation for investigating the relationship of self-efficacy in falling and anxiety with Stroop performance

\begin{tabular}{cccc}
\hline & & Anxiety & Self-Efficacy in Falling \\
\hline \multirow{2}{*}{ Third card } & Correct & $* * 0.318$ & $* * 0.211$ \\
& Reaction & $* *-0.328$ & $* *-282$ \\
& Reaction & 0.009 & $* * 0.311$ \\
Fourth card & Error & $* * 0.386$ & $* * 0.141$ \\
& Correct & $* * 0.203$ & $* 0.111$ \\
& Reaction & $* *-0.196$ & $* * 0.193$ \\
& Reaction & $* * 0.200$ & $* * 0.464$ \\
\hline$* \mathrm{P}<0.01 * \mathrm{P}<0.05$ & Error & $* 0.141$ & $* * 0.251$
\end{tabular}

$\stackrel{\text { Iranan sounanat of }}{A} G \in I N G$

Table 3. Regression results to predict anxiety and self-efficacy in falling based on Stroop's performance

\begin{tabular}{|c|c|c|c|c|c|}
\hline Variable & B & Standard Deviation & & $\mathbf{T}$ & Significance \\
\hline Anxiety & -0.465 & 0.220 & -0.164 & -2.119 & $* * 0.032$ \\
\hline Self-efficacy in falling & 0.507 & 0.089 & 0.448 & 5.232 & $* * 0.000$ \\
\hline
\end{tabular}

scriptive statistics of the variables studied are reported in Table 1.

As observed in Table 1, the highest average score belonged to self-efficacy variable of falling (58.550) and to the amount of reaction to the fourth card (49.472); the fourth card reaction score was 37.2766. Also, the highest standard deviation score corresponding to the score of the fourth card reaction was 78.548, and the fourth card's score was 54.708. The Pearson's correlation test was used to examine the relationship between self-efficacy in falling and anxiety by Stroop test performance. The Kolmogorov-Smirnov test was employed before the Pearson's test in order to assess the normality of the variable. The results did not show any significance. Therefore, the distribution of the variables was normal, and Pearson's test could be used (Table 2).

As seen in Table 2, a significant correlation was established between Stroop's performance (third and fourth card) in all the four sub-scales including errors, integrity, reaction, and interference and anxiety as well as self-efficacy in falling in the elderly. In addition, regression analysis assessed the level of anxiety prediction and self-efficacy in falling based on Stroop test performance. The regression test results are reported in Table 3.

As observed in Table 3, both anxiety and self-efficacy variables of falling can be predicted by Stroop test performance. The results indicated that the performance of the test predicts the anxiety and self-efficacy in falling negatively .

\section{Conclusion}

The Stroop test performance was significantly correlated to anxiety, which was in agreement with the studies by Reiner et al. [7] and Kertz et al. [8]. The results also showed that Stroop performance was significantly correlated to the self-efficacy in falling, which was in agreement with the study by Holford et al. [9] and Walson et al. [10]. The result of the regression test also indicated that Stroop test performance predicted the anxiety and self-efficacy in the falling of the elderly. Therefore, Stroop test performance can be improved in the elderly by conducting training classes and performing different group treatments in order to indirectly reduce the amount of anxiety and increase the self-efficacy in the elderly.

\section{Acknowledgments}

This research did not receive any specific grant from funding agencies in the public, commercial, or not-forprofit sectors.

\section{Conflict of Interest}

The authors declared no conflicts of interest. 


\title{
نقش عملكرد در آزمون استروي در ييشبينى اضطراب وخودكارآمدى در افتادن در سالمندان شهر

\author{
"اسماعيل صدرى دميرجى 'ه، ساناز بهبوئى'، آرزو مجرد' \\ 1- كروه علوم تربيتى، دانشكده علوم تربيتى وروانشناسى، دانشكاه محقق اربيلى، اردبيل، ايران.

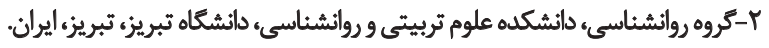

\begin{abstract}
حكيد

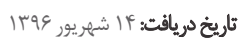

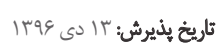

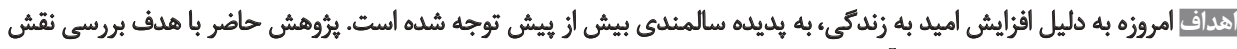

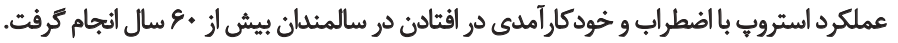

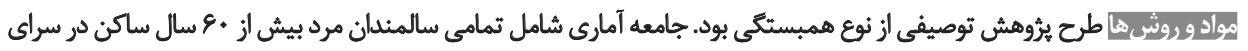

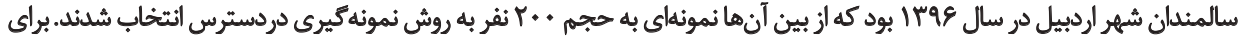

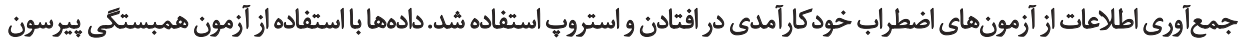
وركرسيون تحليل شد.

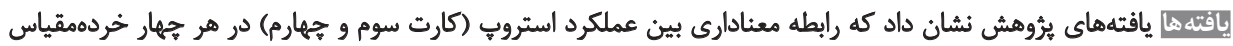

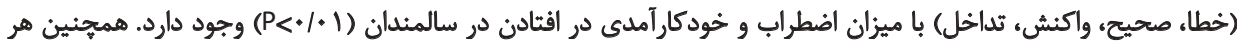

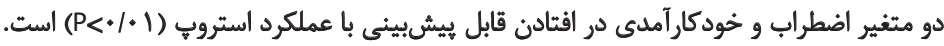

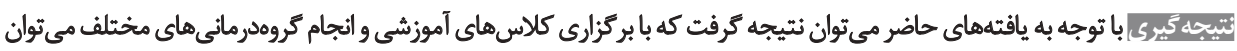

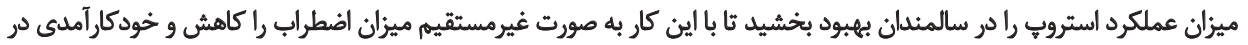
افتادن را در سالمئدان افزايش داد رادي.

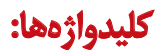

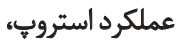

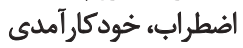

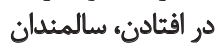

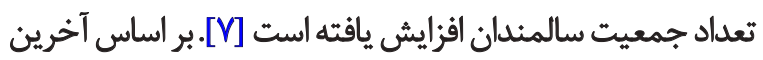

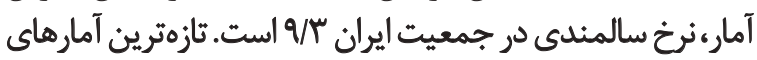

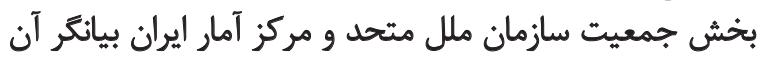

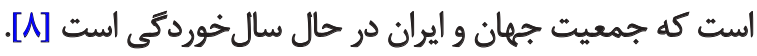

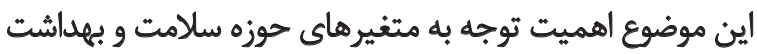

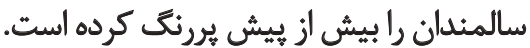

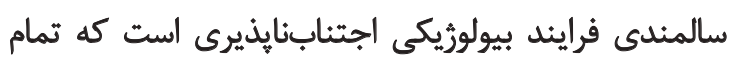

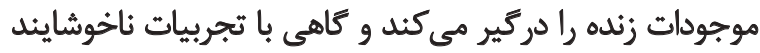

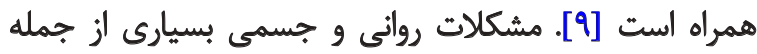

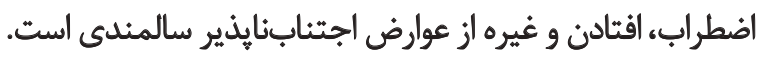

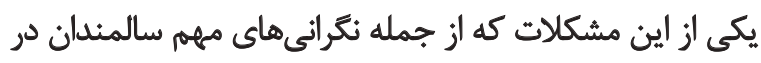

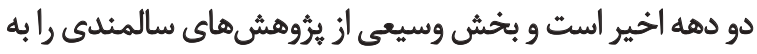

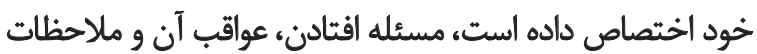

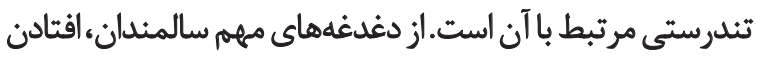
است كه به زمين خوردن ناخواسته فرد منجر مي أشود ودر نتيجها

كنر از دوره سالمندى، البته به شرط بقاه برائ بإئ انسانها

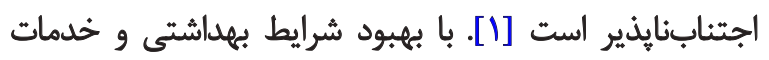

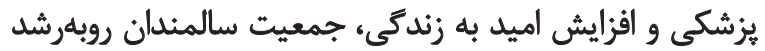

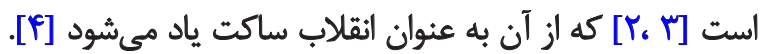

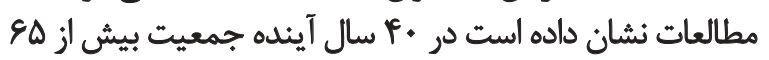

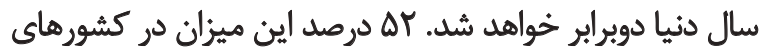

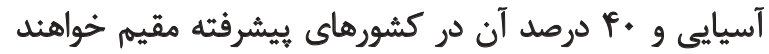

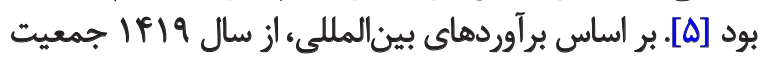

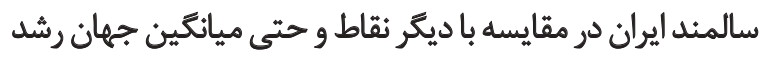

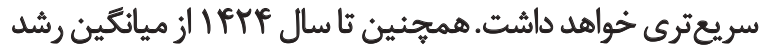

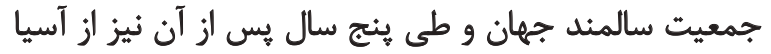
بيشى خواهد كرفت [ع].

در بيشتر كشورهاى جهان سوم، طى نيمقرن اخير نسبت و 
عصبىروانى است كه برخى روانيزشكان و روان كاوان، بخش رونش

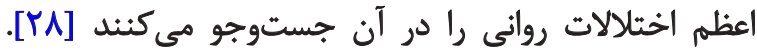

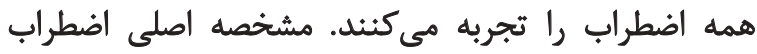

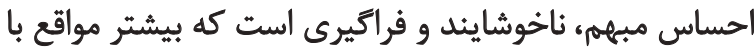

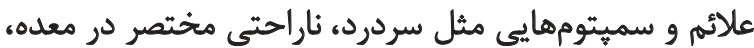

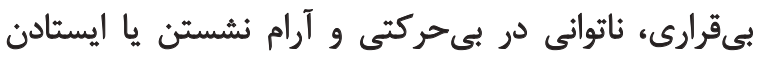

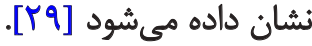

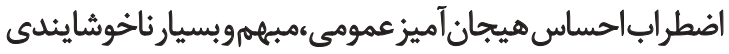

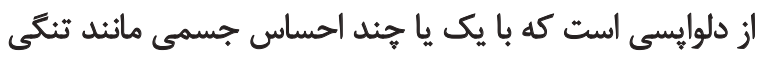

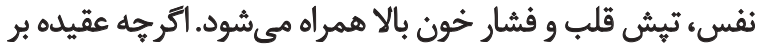

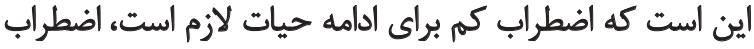

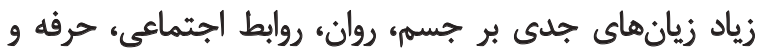

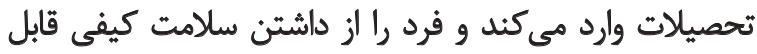

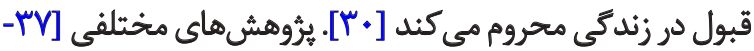

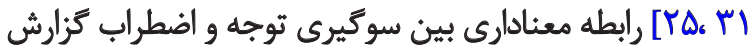

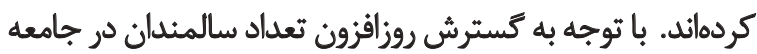

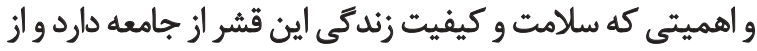

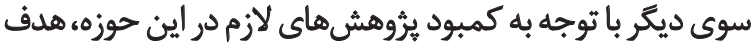

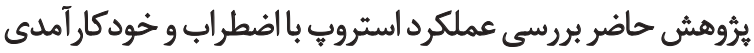
در افتادن در سالمندان بيشتر از •و سال است.

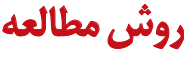

يثروهش حاضر مطالعهايى توصيفى از نوع همبستكى است.

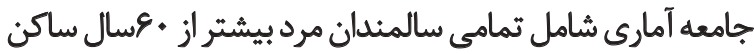

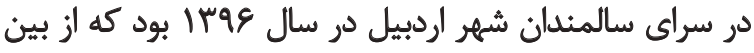

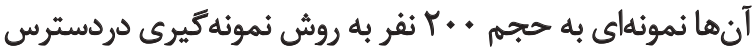

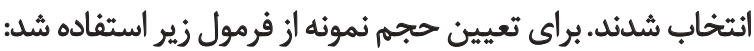

$n=\frac{N z^{2} s^{2}}{N d^{2}+z^{2} s^{2}}$

اين مقاله به لحاظ كميثه اخلاق، به تأييد اداره بهزيستى

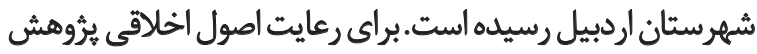

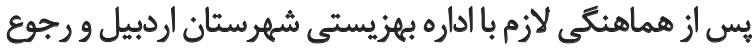

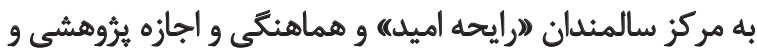

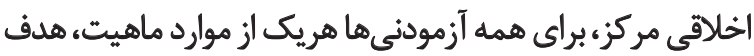

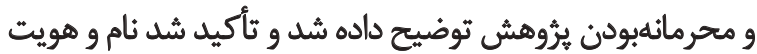

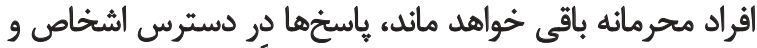

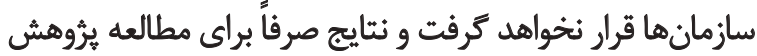

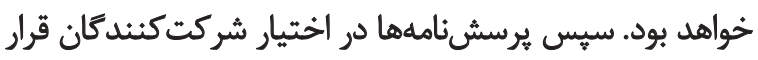

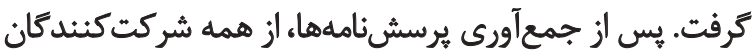

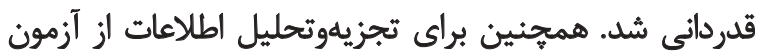

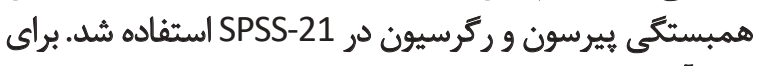
جمع أورى اطلاعات از ابزارهاى زير استفاده شد:
موجب عواملى مانند تحمل ضربه خشن، ازدستدادن هوشيارى

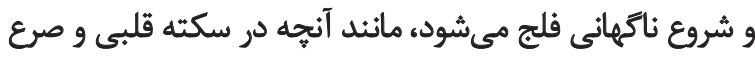

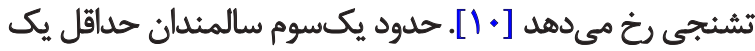

بار افتادن در سال را تجربه مي كنيند [11، II)]

علاوه بر عوامل جسمانى، اجتماعى و فيزيولوزيكى، عوامل

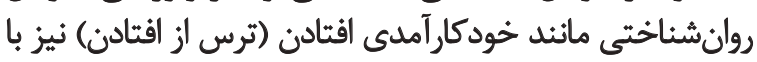

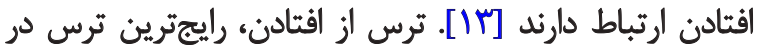

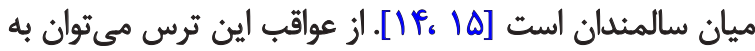

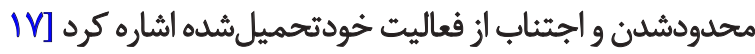

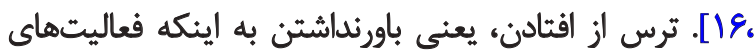

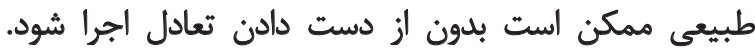

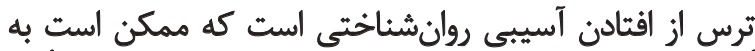

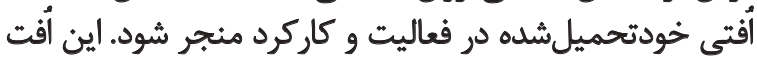

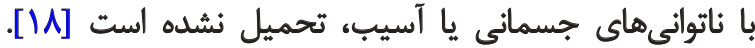

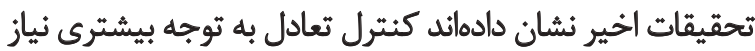

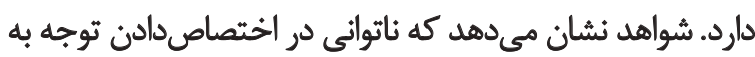

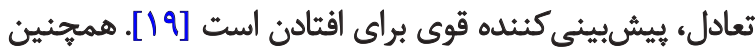

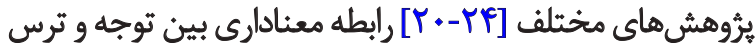

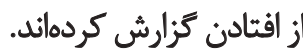

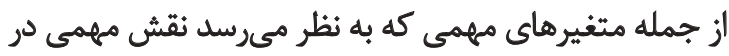

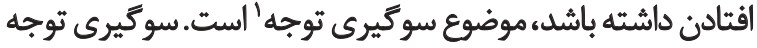

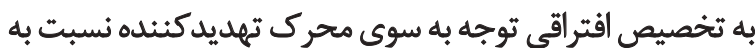

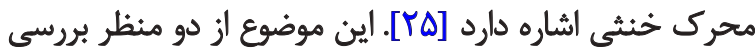

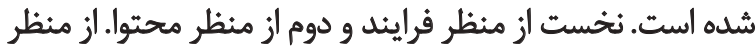

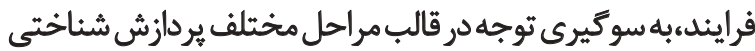

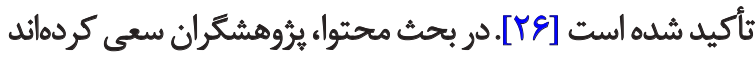

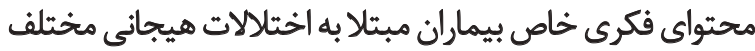

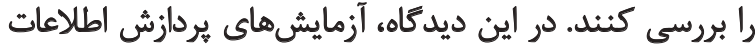

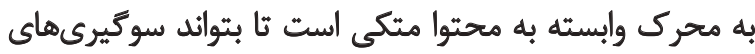

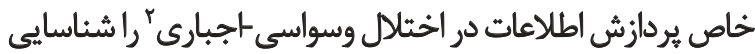

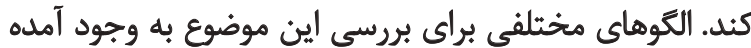

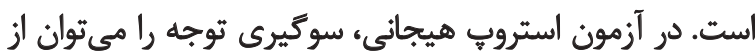

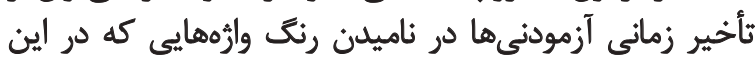

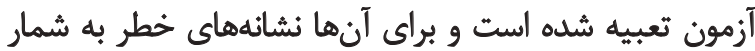

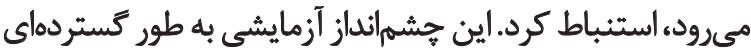

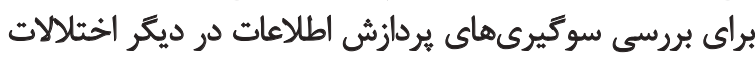

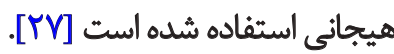

سوكيرى توجه مي تواند با مشكلات بسيارى در سالمندان

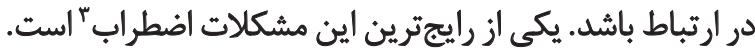

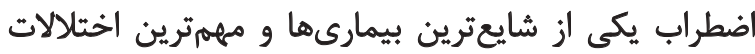

1. Bound attention

2. Obsessive Compulsive Disorder (OCD)

3. Anxiety 


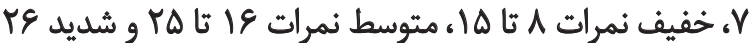

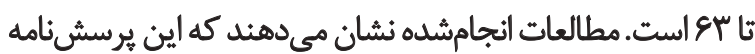

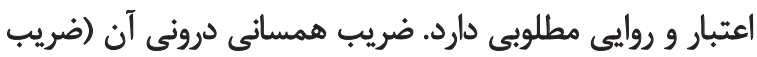

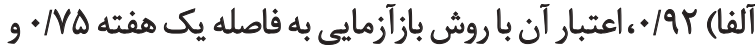

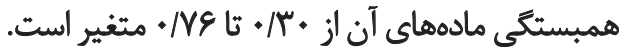

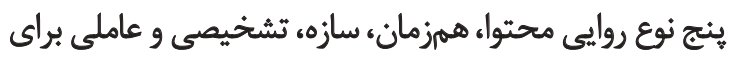

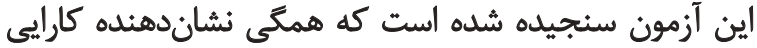

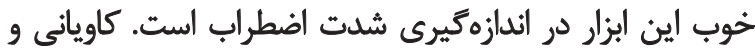

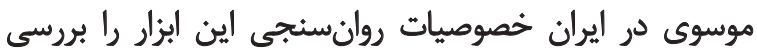

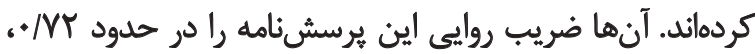

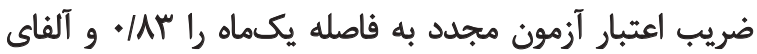

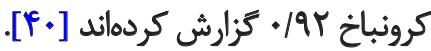

يرسش ئامه خودكار آمدى در افثتادن (FES-I)

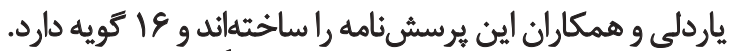

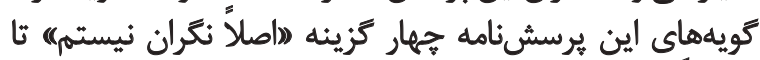

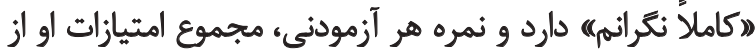

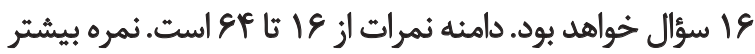

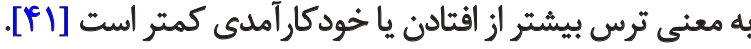

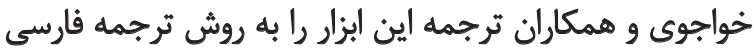

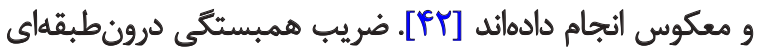

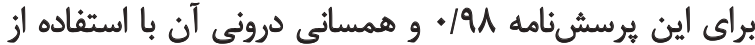

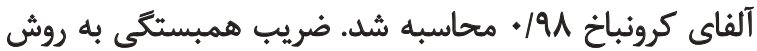

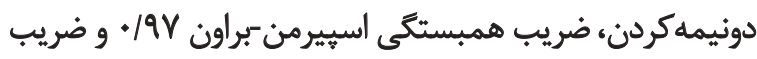

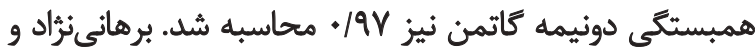

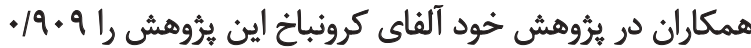

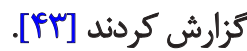

يافتهها

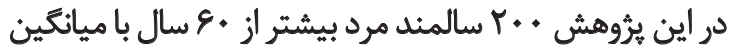

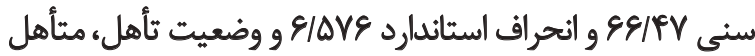
شركت كردند. آمارههاى توصيفى متغيرهاف استيأ تحت مطارد جدول شماره ا كزارش شده است توريفي

همانطور كه در جدول شماره ا مشاهده مى دوداد، بيشترين

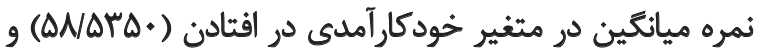

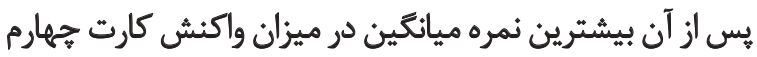
(Fq/FVT)

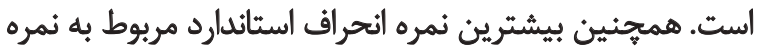

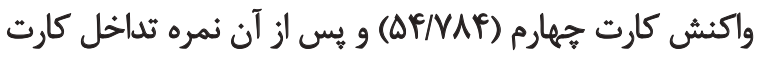

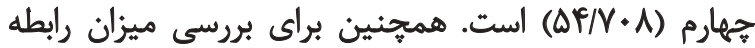

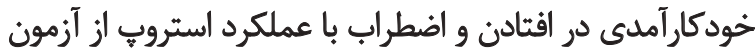

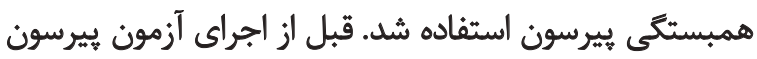

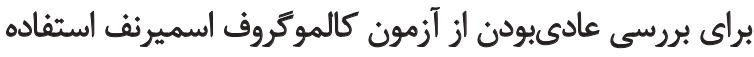

$$
\text { آزمون استروب }
$$

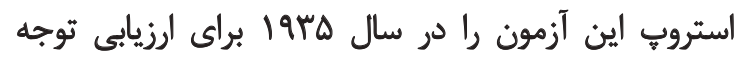

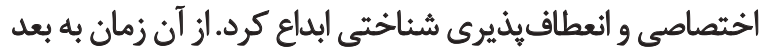

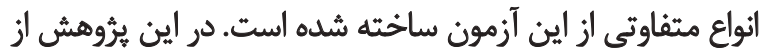

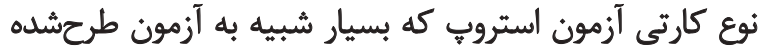

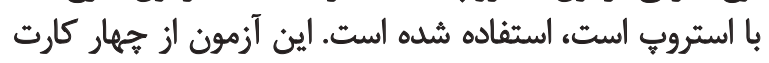

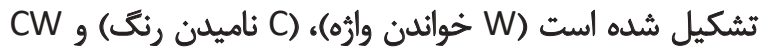

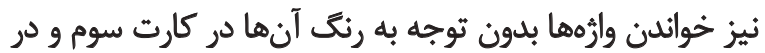

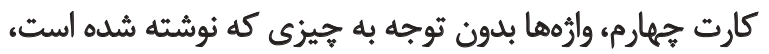

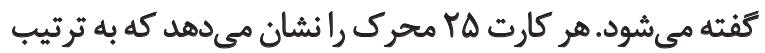

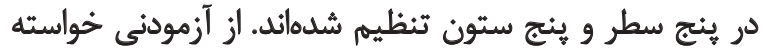

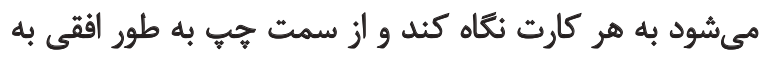

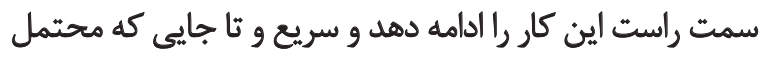

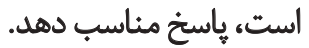

در كارت W محرك، نام رنكهاى ينج

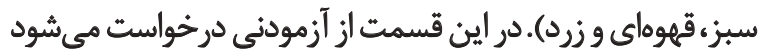

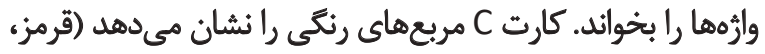

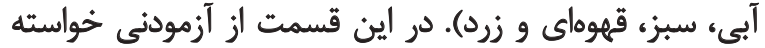

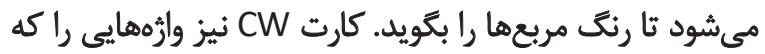

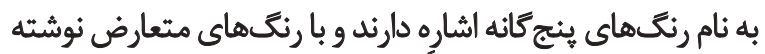

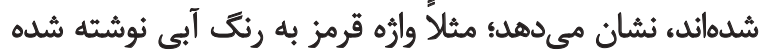

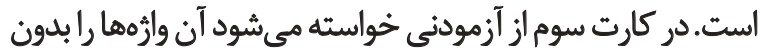

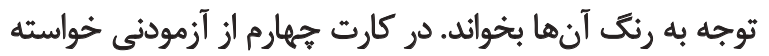

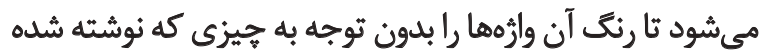

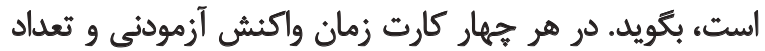

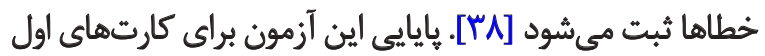

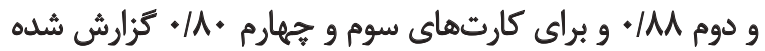

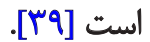

\section{برسش بامه اضطراب بك}

اروين بك" و همكارانش در سال • 199 برسشنامه اضطراب

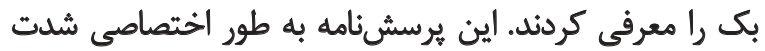

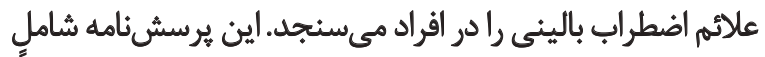

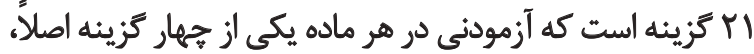

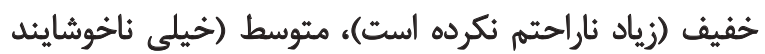

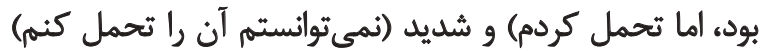

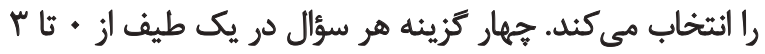

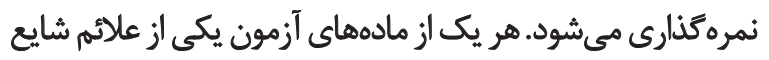

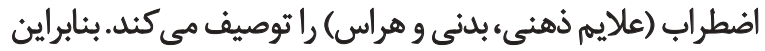

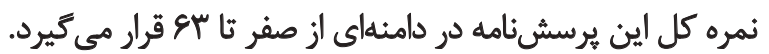

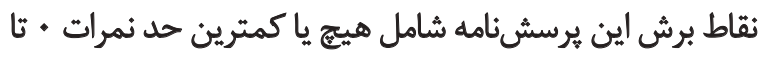

4. Erwin Beck 
جدول أ. آمارههاي توصيفى متغيرهاي ثيروهش

\begin{tabular}{|c|c|c|c|}
\hline انحراف استاندارد & مياتكّين & مؤلفها & \\
\hline f/৭ه内q & QNATL. & خودكارآهدى در افتتادن & \\
\hline $1 . / K 89$ & $|F /| T \mid$ & اضطراب & \\
\hline 佂 &.$/ . r \Delta$ & خطا & \multirow{4}{*}{ كارت اول } \\
\hline.$/ M T Y$ & $r F / Q 9 \Delta$ & صحيح & \\
\hline V/IIT & TY/AID & واكشش & \\
\hline r/Dar &.$/ \Delta V E$ & تناخل & \\
\hline glova & T/DQQ & خطا & \multirow{4}{*}{ كارت دوم } \\
\hline P/MT & $r r / r M$ & صحيح & \\
\hline IW/KeT & $r E / T \cdot \Delta$ & واكنش & \\
\hline 19/AFr & $1.1 . \Delta 9$ & تماخل & \\
\hline 1/Apq & 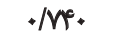 & خط & \multirow{4}{*}{ كارت سوم } \\
\hline I/AFV & re/rPa & صحيح & \\
\hline 1.1 .49 & ro/PAq & واكنش & \\
\hline $1 . / 281$ & gliar & ت تداخل & \\
\hline P/OY & $r / v$. & خط & \multirow{4}{*}{ كارت جهارم } \\
\hline P/QIP & ri/q.. & صحيح & \\
\hline DF/NAF & $\mathrm{Fq} / \mathrm{Fr}$ & واكنش & \\
\hline$\Delta F / V \cdot \Lambda$ & TV/TVES & ت تلاخل & \\
\hline
\end{tabular}

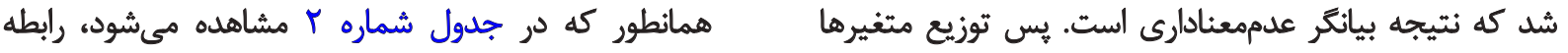

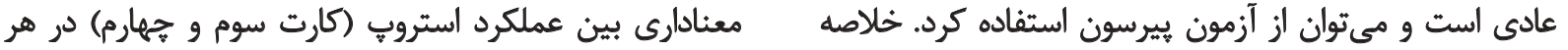

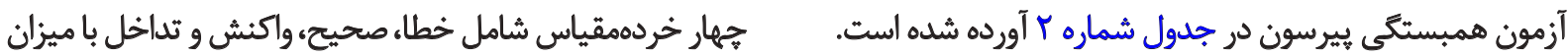

جدول r. نتايج آزمون همبستكى يُرسون براى بررسى رابطه خودكارآمدى در افتادن و اضطراب با عملكرد استروب

\begin{tabular}{|c|c|c|c|}
\hline \multicolumn{2}{|c|}{ خودكارأمدى در الفثتادن } & \multicolumn{2}{|c|}{ اضطراب } \\
\hline$\cdot(M I)^{* *}$ &.$M \mathrm{~A}^{* *}$ & خطا & \\
\hline - /TAY" & $-{ }_{-*} / \mathrm{MT} \Lambda^{* *}$ & صحيح & \\
\hline . $/ 41 I^{* *}$ & $+1++9$ & واكنش & كارت سوم \\
\hline$+\left.|| F\right|^{* *}$ & . RNe*" & تداخل & \\
\hline $.11111^{\circ}$ & $. / r+\mu=t$ & خطا & \\
\hline$-. / 194=$ & $-+/ 1 \varepsilon^{* * *}$ & صحيح & كالت - \\
\hline ./pegen &.$/ r \ldots * *$ & واكثش & نار \\
\hline.$|r \Delta|^{* *}$ &.$/\left.N\right|^{*}$ & ت تداخل & \\
\hline
\end{tabular}


جدول با. تحليل ركرسيون براى بيش بيني اضطراب و خودكار آمدى در افْتادن براساس عملكرد استروب

\begin{tabular}{|c|c|c|c|}
\hline انحراف الستاندارد اندازهكيرى شده & ضريب تعيين تعديل شده & $\mathbf{R}^{\mathbf{r}}$ & $\mathbf{R}$ \\
\hline $10 / V$ & . /Mq &.$/ 14$ & - Mpa \\
\hline
\end{tabular}

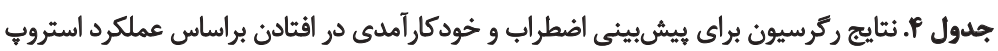

\begin{tabular}{|c|c|c|c|c|c|}
\hline معنى دارى & $\mathbf{T}$ & بتا & انحراف اسثاندارد & B & مثغير \\
\hline$+1+\mu r+*$ & $-4 / 119$ &.$- / 19 F$ &.$/ \pi r$ & $-+/ 480$ & اضطراب \\
\hline$+1++\infty$ & Q/MTT & $+/ 4+\alpha$ & $1+19$ & $+10+V$ & خودكارآملى در افتادن \\
\hline
\end{tabular}

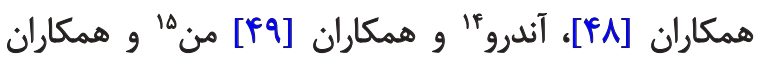

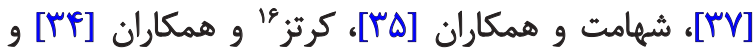

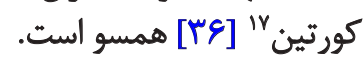

در تبيين نتيجه حاضر ميتوان كفت كله وجود اضطراب به مانه

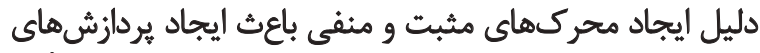

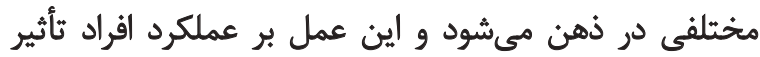

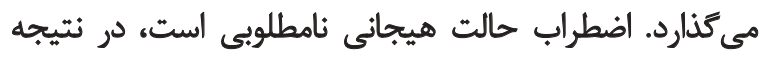

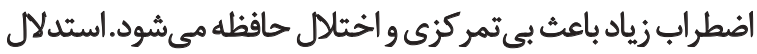

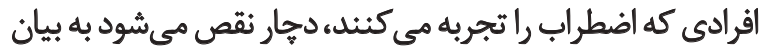

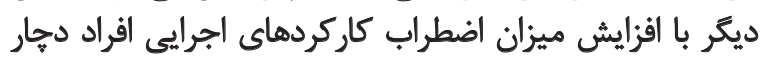

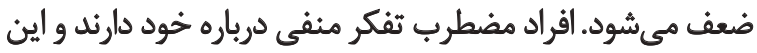

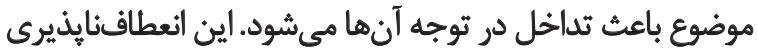

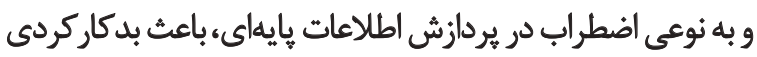

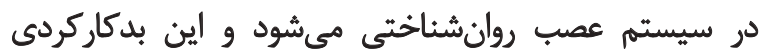

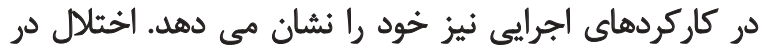

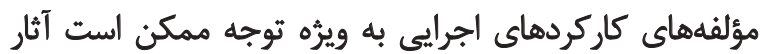

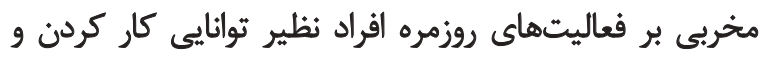

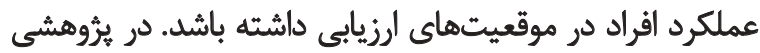

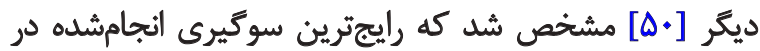

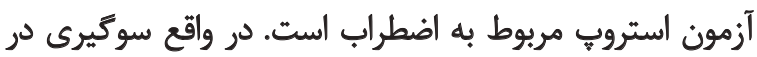

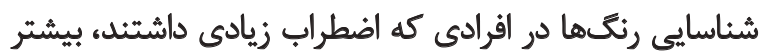

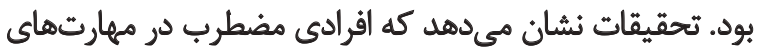

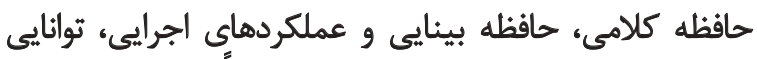

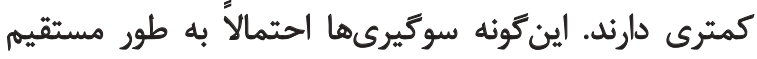

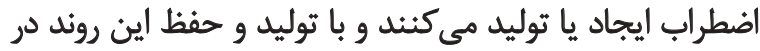

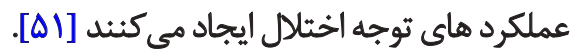

14. Andrew

15. Mann

16. Kertz

17. Curtin
اضطراب و خودكارآمدى در افتادن در سالمندان وجود دارد.

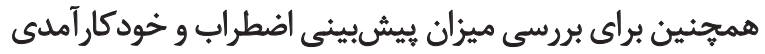

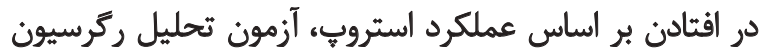

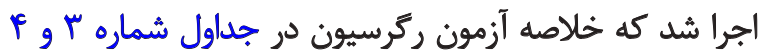
كزارش شده است.

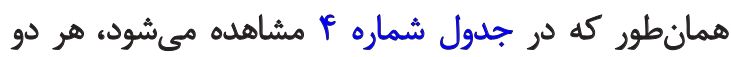

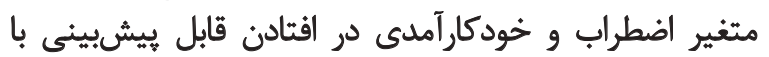

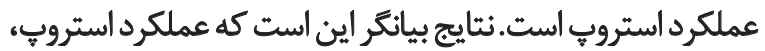

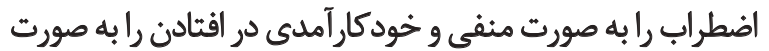
مثبت بيشبينى مئى إند.

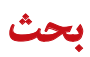

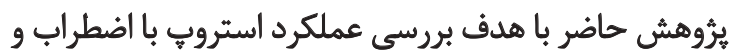

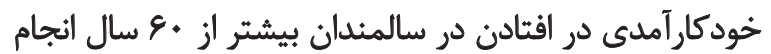

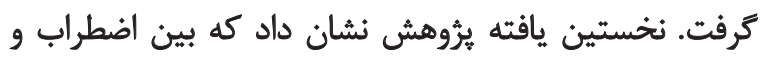

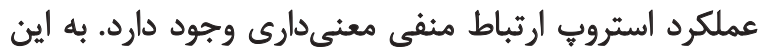

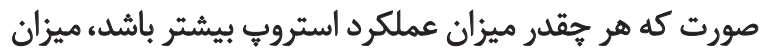

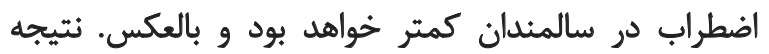

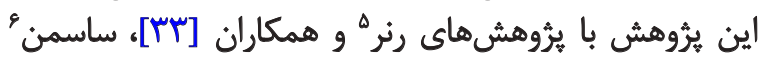

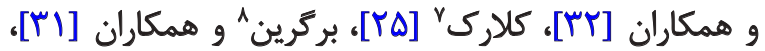

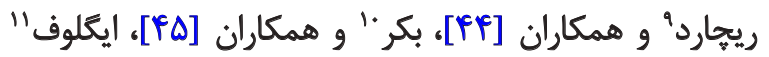

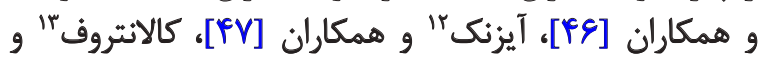

5. Renner

6. Sussman

7. Clark

8. Berggren

9. Richard

10. Becker

11. Egloff

12. Eysenck

13. Kalanthroff 
كاهش مي يابد، در نتيجه به تدريج باعث كاهش خودكارآمدى در

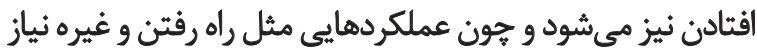

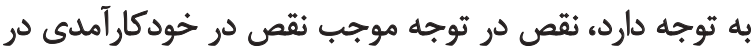

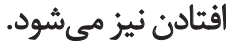

يكى از محدوديتهاي ئروهش حاضر اين بود كه فقط روى

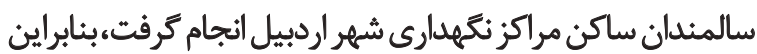

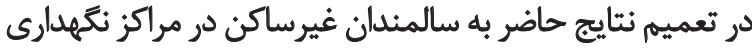

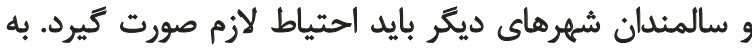

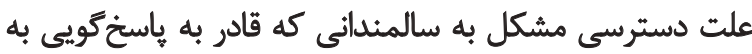

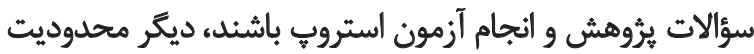

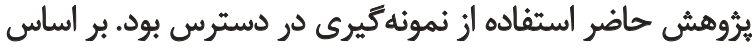

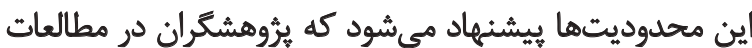

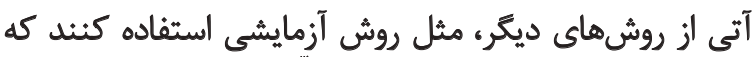

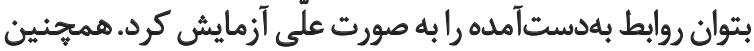

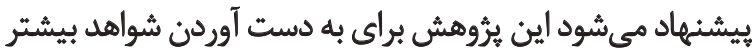
در شهرهاى ديكر نيز انجام شود.

\section{نتيجليَيرى نهايى}

با توجه به يافتهها مىتوان نتيجه كرفت افزايش عملكرد

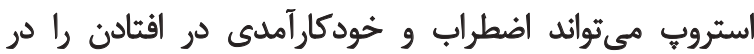

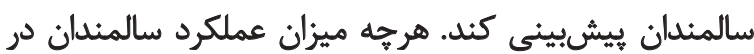

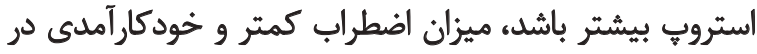

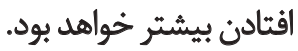

$$
\text { تشكر و قدردائى }
$$

بدينوسيله از همكارى صميمانه مسئول محترم مركز "ارايحه

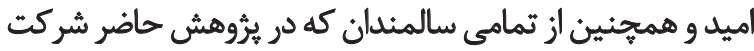
كردند، قدردائى مي وشود. اين مقاله حامى مالمي ندارد.
همجنين در تحقيق ديكرى [f\&] مشخص شد افرادي كه

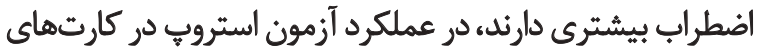

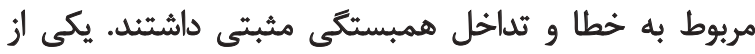

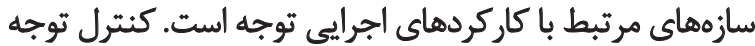

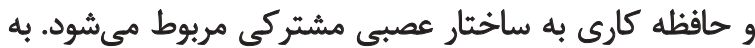

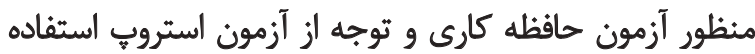

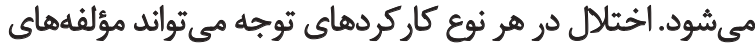

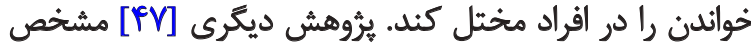

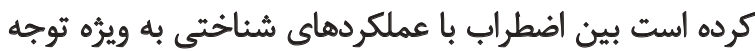

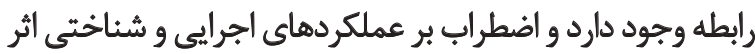

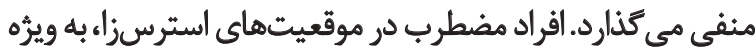

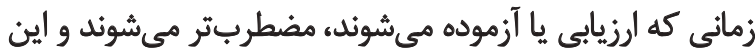

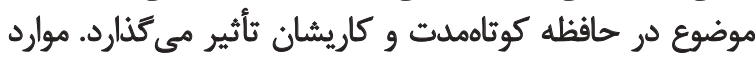

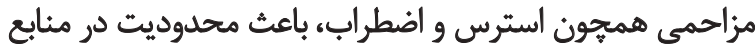

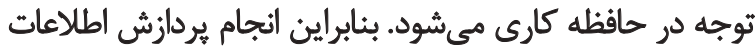

$$
\text { كمتر قابل دسترس است. }
$$

همجنين نتيجه ديكر يُوهش نشان داد بين خودكارآمدى

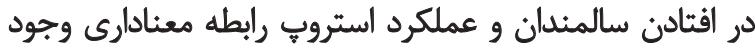

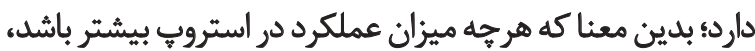

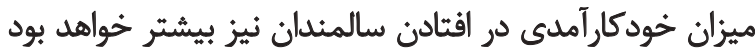

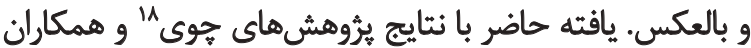

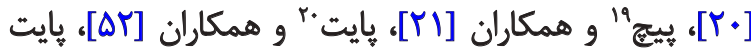

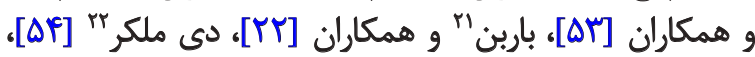

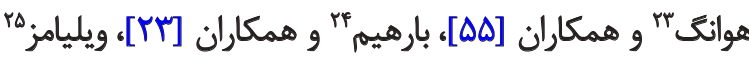

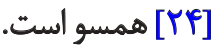

در تبيين يافته حاضر مىتوان جنين كفت كه روند سالمندى

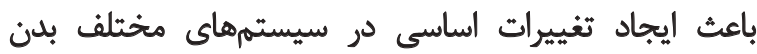

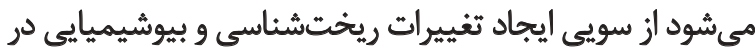

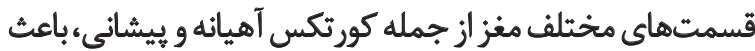

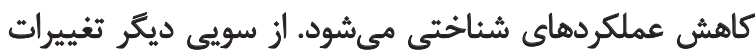

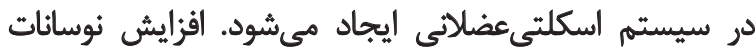

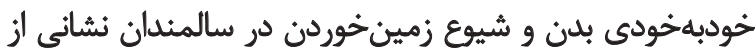

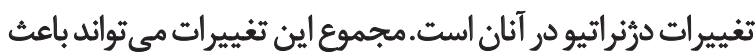

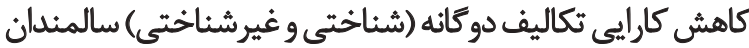

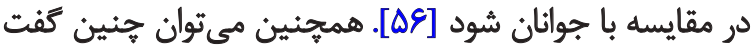

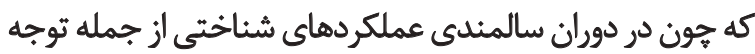

18. Choi

19. Peach

20.Payette

21. Barban

22. De Melker

23. Hoang

24. Bar-Haim

25. Williams 


\section{References}

[1] Saydshohadai M, Heshmat S, Seidfatemi N, Haghani H, Mehrdad N. [The spiritual health of seniors living in sanitarium and home residents (Persian)]. Iran Journal of Nursing. 2013; 26(81):11-20

[2] Lashkarboloki F, Aryaei M, Djazayery A, Eftekhar-Ardebily H, Minaei M. [Association ofdemographic, socio-economic features and some health problems with nutritional status in elderly (Persian)]. Journal of Nutrition Sciences \& Food Technology. 2015; 9(4):27-34.

[3] Hajiaziz A H, Bahmani B, Mahdi N, Manzari Tavakoli V, Barshan A. [Effectivness of group logotherapy on death anxiety and expectancy of elderly living in boarding houses in Kerman (Persian)]. Iranian Journal Ageing. 2017; 12(2):220-31.

[4] Azadchehr M, Rahgozar M, Karimloo M, Adib Haj Bageri M. [To identify some factors effective on survival of the elderly living in nursing home using Copula Competing Risk Model: Bayesian approach (Persian)]. Journal of Health Promotion Management. 2014;3(4):46-55

[5] World Health Organization. Definition of an older or elderly person, health statistics \& health information. Geneva: World Health Organization; 2014

[6] Ahrari S, Moshki M, Bahrami M. [The relationship between social support and adherence of dietary and fluids restrictions among hemodialysis patients in Iran]. Journal of Caring Sciences. 2014; 3(1):11-9. doi: 10.5681/jcs.2014.002

[7] Gilasi H R, Sori H, Yazdani Sh, Taheri Tnjati P. [Methodological considerations in the elderly study: Case study (Persian)]. Iranian Journal of Special Epidemiology. Iranian Journal of Epidemiology 2015; 11(2):1-12.

[8] Feizabadi M, Nakhoda M, Delbari A. [Rresearch databases and geriatrics and gerontology journals: the comparison of databases and their covered journals (Persian)]. Salmand. 2016; 11(2):358-69.

[9] Rashidi Fakari F, Azimi Hashemi M, Rashidi Fakari F. A Qualitative research: Postmenopausal women's experiences of abuse. Procedia - Social and Behavioral Sciences. 2013; 82:57-60. doi: 10.1016/j.sbspro.2013.06.224

[10] Wijlhuizen GJ, de Jong R, Hopman-Rock M. Older persons afraid of falling reduce physical activity to prevent outdoor falls. Preventive Medicine. 2007; 44(3):260-4. doi: 10.1016/j. ypmed.2006.11.003

[11] Khajavi D. [Validation and reliability of Persian version of fall efficacy scale-international (FES-I) in community-dwelling older adults (Persian)]. Salmand. 2013; 8(29):39-47.

[12] Evitt C, Quigley P. Fear of falling in older adults: A guide to its prevalence, risk factors, and consequences. Rehabilitation Nursing. 2004; 29(6):207-10. PMID: 15597999

[13] Sharaf A, Ibrahim H. Physical and psychosocial correlates of fear of falling among older adults in assisted living facilities. Journal of Gerontological Nursing. 2008; 34(12):27-35. doi: 10.3928/00989134-20081201-07

[14] Arfken CL, Lach HW, Birge SJ, Miller JP. The prevalence and correlates of fear of falling in elderly persons living in the community. American Journal of Public Health. 1994; 84(4):565-70. doi: 10.2105/ajph.84.4.565
[15] Ziilstra GAR, Van Haastregt JCM, Van Eijk JTM, Van Rossum E, Stalenhoef PA, Kempen GIJM. Prevalence and correlates of fear of falling, and associated avoidance of activity in the general population of community-living older people. Age and Ageing. 2007; 36(3):304-9. doi: 10.1093/ageing/afm021

[16] Tinetti ME, Richman D, Powell L. [Falls efficacy as a measure of fear of falling]. Journal of Gerontology. 1990; 45(6):P239-P243. doi: 10.1093/geronj/45.6.p239

[17] Iranmanesh H, Arab Ameri A, Sheikh M, Iranmanesh H. [The effect of 2 types of dual-task training on the balance of older adults: Allocated attention ability (Persian)]. Salmand. 2016; 11(1):30-43

[18] Hallford D. J, Nicholson G, Sanders K, McCabe M.P. The association between anxiety and falls: A meta-analysis. The Journals of Gerontology Series B: Psychological Sciences and Social Sciences. 2017; 72(5):729-41. doi: 10.1093/geronb/gbv160

[19] Wollesen B, Schulz S, Seydell L, Delbaere K. Does dual task training improve walking performance of older adults with concern of falling? BMC Geriatrics. 2017; 17(1):213. doi: 10.1186/ s12877-017-0610-5

[20] Choi K, Jeon G-S, Cho S. Prospective study on the impact of fear of falling on functional decline among community dwelling elderly women. International Journal of Environmental Research and Public Health. 2017; 14(5):469. doi: 10.3390/ijerph14050469

[21] Peach T, Pollock K, van der Wardt V, das Nair R, Logan P, Harwood RH. Attitudes of older people with mild dementia and mild cognitive impairment and their relatives about falls risk and prevention: A qualitative study. Plos One. 2017; 12(5):e0177530. doi 10.1371/journal.pone. 0177530

[22] Barban F, Annicchiarico R, Melideo M, Federici A, Lombardi $\mathrm{M}$, Giuli S, et al. Reducing fall risk with combined motor and cognitive training in elderly fallers. Brain Sciences. 2017; 7(2):19. doi: 10.3390/brainsci7020019

[23] Bar-Haim Y, Lamy D, Pergamin L, Bakermans-Kranenburg MJ, van IJzendoorn $\mathrm{MH}$. Threat-related attentional bias in anxious and nonanxious individuals: A meta-analytic study. Psychological Bulletin. 2007; 133(1):1-24. doi: 10.1037/0033-2909.133.1.1

[24] Williams JMG, Mathews A, MacLeod C. The emotional Stroop task and psychopathology. Psychological Bulletin. 1996; 120(1):324. doi: 10.1037/0033-2909.120.1.3

[25] Clark DA. Cognitive-behavioral therapy for OCD. New York: Guilford; 2007.

[26] Koshan M, Vaghe'i S. [Psychiatric nursing (Persian)]. Tehran: Andishe-ye Rafi'; 2011

[27] Sadock B, Sadock V, Ruiz P. Kaplan Sadock's synopsis of psychiatry: Behavioral sciences. [M. Ganji, Persian Trans]. Tehran: Savalan; 2015.

[28] Katzelnick DJ, Kobak KA, DeLeire T, Henk HJ, Greist JH, Davidson JRT, et al. Impact of generalized social anxiety disorder in managed care. American Journal of Psychiatry. 2001; 158(12):1999-2007. doi: 10.1176/appi.ajp.158.12.1999

[29] Yiend J. The effects of emotion on attention: A review of attentional processing of emotional information. Cognition \& Emotion 2010; 24(1):3-47. doi: 10.1080/02699930903205698

[30] Fox E, Russo R, Bowles R, Dutton K. Do threatening stimuli draw or hold visual attention in subclinical anxiety? Journal of 
Experimental Psychology: General. 2001; 130(4):681-700. doi: 10.1037/0096-3445.130.4.681

[31] Berggren N, Derakshan N. Attentional control deficits in trait anxiety: Why you see them and why you don't. Biological Psychology. 2013; 92(3):440-6. doi: 10.1016/j.biopsycho.2012.03.007

[32] Sussman TJ, Jin J, Mohanty A. Top-down and bottom-up factors in threat-related perception and attention in anxiety. Biological Psychology. 2016; 121:160-72. doi: 10.1016/j.biopsycho.2016.08.006

[33] Renner KA, Valentiner DP, Holzman JB. Focus-of-attention behavioral experiment: an examination of a therapeutic procedure to reduce social anxiety. Cognitive Behaviour Therapy. 2016; 46(1):60-74. doi: 10.1080/16506073.2016.1225814

[34] Kertz SJ, Stevens KT, Klein KP. The association between attention control, anxiety, and depression: the indirect effects of repetitive negative thinking and mood recovery. Journal of Anxiety, Stress \& Coping. 2017; 30(4):456-468. doi: 10.1080/10615806.2016.1260120

[35] Shahamat DehSorkh F, Salehi Fadrdari J. [attentional bias in state and trait anxiety: A dot-probe study (Persian)]. Journal of Advanced Psychological Research. 2013; 8(29):183-194.

[36] Curtin A. Prevention of falls in older adults. Medicine and Health. 2005; 88(1):22-5. PMID: 15779552

[37] Mann R, Birks Y, Hall J, Torgerson D, Watt I. Exploring the relationship between fear of falling and neuroticism: a cross-sectional study in community-dwelling women over 70. Age and Ageing. 2005; 35(2):143-7. doi: 10.1093/ageing/afj013

[38] Rezaei Niyasr A, Zare H, Barjesteh F. [Assessment of cognitive eunction in obese \& overweight children compared with children of normal weight in the Tower of London \& Stroop test (Persian)] Health Psychology. 2017; 6(2):35-50.

[39] Basharpour S. [The study of information processing speed, automated and controlled processing, and the effect of antidepressants on these three variables in depression disorder (Persian)] [MSc. thesis]. Ardabil: University of Mohaghegh Ardabili; 2004.

[40] Kaviani H, Mousavi AS. [Psychometric properties of the Persian version of Beck Anxiety Inventory (BAI) (Persian)]. Tehran University Medical Journal. 2008; 66(2):136-140.

[41] Yardley L, Beyer N, Hauer K, Kempen G, Piot-Ziegler C, Todd C. Development and initial validation of the Falls Efficacy ScaleInternational (FES-I). Age and Ageing. 2005; 34(6):614-9. doi: 10.1093/ageing/afi196

[42] Khajavi D, Farokhi A, Jaberi Moghadam AA, Kazemnejad A. [The impact of a training intervention program on fall-related psychological factors among male older adults in Arak (Persian)]. Salmand. 2014; 9(1):32-39.

[43] Borhani Nezhad VR, Rashedi V, Tabe R, Delbari A, Ghasem zade $\mathrm{H}$. [Relationship between fear of falling and physical activity in older adults (Persian)]. Medical Journal of Mashhad University of Medical Sciences. 2015; 58(8):446-52.

[44] Richards A, French CC, Johnson W, Naparstek J, Williams J. Effects of mood manipulation and anxiety on performance of an emotional Stroop task. British Journal of Psychology. 1992; 83(4):479-91. doi: 10.1111/j.2044-8295.1992.tb02454.x
[45] Becker ES, Rinck M, Margraf J, Roth WT. The emotional Stroop effect in anxiety disorders. Journal of Anxiety Disorders. 2001 15(3):147-59. doi: 10.1016/s0887-6185(01)00055-x

[46] Egloff B, Hock M. Interactive effects of state anxiety and trait anxiety on emotional Stroop interference. Personality and Individual Differences. 2001; 31(6):875-82. doi: 10.1016/s01918869(00)00188-4

[47] Eysenck MW, Derakshan N, Santos R, Calvo MG. Anxiety and cognitive performance: Attentional control theory. Emotion. 2007 7(2):336-53. doi: 10.1037/1528-3542.7.2.336

[48] Kalanthroff E, Henik A, Derakshan N, Usher M. Anxiety, emotional distraction, and attentional control in the Stroop task. Emotion. 2016; 16(3):293-300. doi: 10.1037/emo0000129

[49] Du Rocher AR, Pickering AD. Trait anxiety, infrequent emotional conflict, and the emotional face Stroop task. Personality and Individual Differences. 2017; 111:157-62. doi: 10.1016/j. paid.2017.02.017

[50] Williams JMG, Mathews A, MacLeod C. The emotional Stroop task and psychopathology. Psychological Bulletin. 1996; 120(1):324. doi: $10.1037 / 0033-2909.120 .1 .3$

[51] Goriian F, Abdollahi M H.. [Comparison of executive functions and the cognitive estimation and interpretation bias in patients with social anxiety disorder and normal individuals (Persian)]. Journal of Cognitive Psychology. 2016; 4(3):41-50.

[52] Payette MC, Bélanger C, Léveillé V, Grenier S. Fall-related psychological concerns and anxiety among community-dwelling older adults: Systematic review and meta-analysis. PLOS ONE 2016; 11(4):e0152848. doi: 10.1371/journal.pone.0152848

[53] Payette MC, Bélanger C, Benyebdri F, Filiatrault J, Bherer L, Bertrand JA, et al. The association between generalized anxiety disorder, subthreshold anxiety symptoms and fear of falling among older adults: preliminary results from a pilotstudy. Clinical Gerontologist. 2017; 40(3):197-206. doi: 10.1080/07317115.2017.1296523

[54] De Melker Worms JLA, Stins JF, van Wegen EEH, Loram ID, Beek PJ. Influence of focus of attention, reinvestment and fal history on elderly gait stability. Physiological Reports. 2017 5(1):e13061.doi: 10.14814/phy2.13061

[55] Hoang OTT, Jullamate P, Piphatvanitcha N, Rosenberg E. Factors related to fear of falling among community-dwelling older adults. Journal of Clinical Nursing. 2016; 26(1-2):68-76. doi 10.1111/jocn.13337

[56] Babapour M, Raheb G, Eglima M. [The relationship between social support and life satisfaction among elderly nursing home residents in Tehran (Persian)]. Salmand. 2014; 9(1):6-13. 
